\title{
Zur Kenntniss und Trennung der Platinerz-Metalle*).
}

1. Aufschliessen der Erze.

Es sind im Laufe der Zeit von den verschiedenen Forschern, welche sich mit Untersuchungen über die Gruppe der Platinmetalle beschäftigt haben, bekanntlich die verschiedensten Methoden zur Aufschliessung der Erze empfohlen worden. Als beste Methode muss natürlich diejenige angesehen werden, welche mit dem geringsten Aufwand an Zeit und Kosten die grösste Ausbeute an reinen Metallen liefert.

Gleichzeitig haben nun einerseits Gibbs**), durch die Probiranstalt der Vereinigten Staaten und die Münze in Philadelphia in den Besitz von etwa $600 \mathrm{Grm}$. sibirischen Osmiridiums gelangt, andererseits Claus***) sich die verdienstliche Aufgabe gestellt, sämmtliche bisher in Vorschlag gekommene Methoden zur Aufschliessung und Trennung der Platinmetalle auf ihre Vorzüglichkeit za prüfen und zu entscheiden, welche dieser Methoden als die zweckmässigste angesehen werden müsse.

Die älteste Methode rührt von $\mathrm{V}$ a uquelin her, und besteht in einem Schmelzen des Osmiridiums mit Salpeter; Berzelius hat dieselbe mit gutem Erfolge angewendet. Wollaston hat an Stelle des Salpeters Aetzkạli angewendet, doch ist diese Modification minder zweckmässig als die erste.

Claus $\dagger$ ) vereinigte beide Methoden, er schmelzte nämlich das Erz mit 1 Theil Aetzkali and 2 Theilen Salpeter auf 1 Theil Erz. Während nun $\mathrm{Claus}$ seine Methode für die beste erklärt, welche bisher zur Anwendang gekommen ist, macht Gibbs an derselben einige Ausstellungen. Da die Methode von $\mathrm{Claus}$ von den meisten Chemikern noch fortwährend angewandt wird und wohl auch die allgemeinste Anwendung gestattet, so soll sie im Nachstehenden mit des Verfassers eigenen Worten angeführt werden. Von der russischen Regierung hatte der Verf. $420 \mathrm{Grm}$. des auserlesensten sibirischen Osminm-Iridiums in Schuppenform erhalten, deren Aufschliessung er folgendermaassen beschreibt:

*) Die Bearbeitung dieses Abschnittes des Berichtes hat auf meinen Wunsch Herr Dr. A. Fo rster ausgeführt. R. F.

**) American. Journ. of Science and Arts. 2 Ser. vol. XXXI; Journ. f. prakt. Chem. 84, p. 65.

***) Journ. f. prakt. Chem. 85, p. 121-161.

$\dagger$ Ebendaselbst 85, p. 143 u. 145, 
„,90 Grm. Erz wurden mit $180 \mathrm{Grm}$. Salpeter und $90 \mathrm{Grm}$. Aetzkali in einem silbernen Tiegel von einem Liter Capacität ein bis anderthalb Stunden bindurch einer starken Rothglühhitze ausgesetzt. Um den Tiegel vor dem Schmelzen zu schützen, wurde er in einen hessischen Tiegel gethan, dessen Boden einen halben Zoll hoch mit Magnesia überdeckt war. Die glühend schmelzende Flüssigkeit wurde vorsichtig von dem unangegriffenen Erze, dessen Menge bedeutend abgenommen hatte, in eine Schale von Eisenblech ausgegossen. Der Tiegel wurde abermals mit der oben angegebenen Menge Erz und Schmelzmittel beschickt and die Operation so oft wiederholt, bis alles Erz zur Anwendung gekommen war. Nach fünf Schmelzungen war das so weit beendigt, aber es blieb ein namhafter Rest unaufgeschlossenen Erzes übrig, welcher nach zwei bis drei Schmelzungen, mit dex angegebenen Quantität der Agentien behandelt, einen nicht sehr ansehnlichen Rest von weniger als $30 \mathrm{Grm}$. unaufgeschlossen zurückliess, welcher nicht mehr bearbeitet, sondern für spätere Operationen aufbewahrt wurde. Die Schmelzkuchen wurden durch einen starken Schlag aus den Schalen geschlagen, gröblich gepulvert und in einer verschliessbaren Flasche mit 14 Liter destillirten Wassers tüchtig bis zum Auflösen geschüttelt und an einen dunkeln Ort zum Abklären hingestellt. Nach Verlauf von vier Stunden wurde die geklärte Lösung mittelst eines Glashebers von dem schwarzen pulverförmigen Bodensatze abgehoben. Der Bodensatz wurde mit Wasser vermischt anf einen Glastrichter gegeben, dessen Röhre mit einem Asbestpfropf versehen war, und gut ausgesüsst.

Auf diese Weise wurde eine Lösung $A$ und ein schwarzes Pulver $B$, die aufgeschlossenen Oxyde der verschiedenen Platinmetalle, erhalten.

A war sehr stark gefärbt, tief orangefarben, in dicken Schichten fast undurchsichtig, roch nur schwach nach Osmiumsäure und enthielt freies Kali, salpetrigsaures, osmigsaures, ruthensaures und salpetersaures Kali, etwas freie Osmiumsäure und sonst keine Spur anderweitiger Platinmetalle. Die Lösung wurde, wie später gezeigt werden soll, zur Darstellung der Ruthenverbindungen und der Osmiumsäure benutzt.

B war sammtsehwarz, pulverförmig, krystallinisch and bestand der grössten Masse nach aus Iridiumoxyd und saurem iridiumsaurem Kali, mit Antheilen von Ruthensesquioxydul und Osmiumoxyd, Eisenoxyd, Spuren Kupferoxyd und Palladiumoxyd, welche sämmtlich in Säuren löslich sind, ferner aus einem in Säuren nnlöslichen Reste von Oxyden des Iridiums, Platins und Rhodiums and endlich aus geringen An- 
theilen unaufgeschlossenen Erzes. Es wurde B einer nochmaligen Schmelzung unterworfen, um das Ruthen auszuziehen und zwar das Oxydgemenge und die Schmelzmittel wieder in dem oben angegebenen Verhältnisse. Mit vier Schmelzungen reicht man hier aus. Man erhält nun auf diese Weise wieder eine Lösung A, welche in Beziehung ihres Concentrationszustandes, der Intensität, der Farbe und ihres Gehaltes an Ruthen dem ersten Auszuge nur wenig nachgibt, und den früheren schwarzen Rest B, welcher noch nicht von allem Buthen erschöpft ist, sondern ungefähr den vierten Theil der im Erze enthaltenen Menge desselben zurückhält; aber es ist nicht rathsam, ihm zum dritten Male durch Schmelzmittel das Ruthen entziehen zu wollen."

Soweit die Aufschliessung nach der Methode Clans'. Die weitere Trennung soll in einem späteren Abschnitte noch besprochen werden.

Gibbs *) theilt mit, dass er bei Befolgen dieser Methode nicht so gute Resultate als Cla us erhalten habe, so blieben bei 2 Schmelzungen von $500 \mathrm{Grm}$. californischen Osmiridiums $300 \mathrm{Grm}$. unaufgeschlossen. Vorübergehendes Schmelzen des rohen Erzes mit kohlensaurem Natron, Auslaugen und Auswaschen mit Wasser, um die Kieselsäure und andere Verunreinigungen zu entfernen, fand Gibbs sehr wesentlich für ein gutes Gelingen des Processes.

Fritzsche und Struve ${ }^{* *}$ ) ersetzen den Salpeter durch chlorsaures Kali and schmelzen demnach das Erz mit gleichen Theilen Kalihydrat und chlorsaurem Kali, wobei kein merkbares Entweichen von Osmiumsäure stattfindet. Die Temperatur darf nicht hoch steigen, und das starke Schäumen der Masse erfordert grosse Gefässe. Aus diesem Grunde und wegen der grösseren Kostspieligkeit zieht Gibbs das Verfahren von Claus dem eben beschriebenen vor. Claus selbst ist der Ansicht, dass das Schmelzen mit chlorsaurem Kali unter Umständen seine Vorzüge haben möge, dass aber der Salpeter jedem andern Oxydationsmittel vorzuziehen sei, weil er in der Glühhitze, wo die Einwirkung der Agentien auf das Erz am grössten ist, seinen Sauerstoff nur langsam verliert, und weil er eine leichtflüssige Schmelze bildet. welche die aufgeschlossenen Antheile des Erzes abspült und so das unangegriffene Erz der Einwirkung der Agentien blosslegt; ferner weil

*) Americ. Journ. of Science and Arts. 2. Ser. vol. XXXI; Journ. f, prakt. Chem. 84. 66 .

**) Journ. f. prakt. Chem. 37. 483 , 
die leichtflüssige Schmelze mit grosser Bequemlichkeit durch Ausgiessen ohne Verlust aus dem Tiegel entfernt werden kann und man nicht, wie bei Anwendung von chlorsaurem Kali durch Aufschäumen einen Verlust an kostbarem Material zu befiurchten hat. Endlich verliert das chlorsaure Kali, wie das Baryumbyperoxyd und der salpetersaure Baryt zu leicht seinen Sauerstoff, viel früher als in starker Glühhitze die energische Aufschliessung durch Oxydation beginnt.

Fremy*) sucht das Erz durch Rösten im Sanerstoffstrom von der überwiegenden Menge Osmiamsäure zu befreien, darauf schmelzt er die zurückbleibende Masse mit Salpeter und destillirt aus ihr mittelst Salpetersäure den Rest der Osmiumsäure ab. Gibbs erhielt bei Anwendung dieser Methode nur gute Resultate, wenn das ursprüngliche Erz sehr reich an Osmium war, im anderen Fall wurde durch den Röstprocess nur wenig Osmiumsäure ausgetrieben, und hält es der Verf. dann für zweckmässiger, sofort nach der von $\mathrm{Claus}$ empfohlenen Methode aufzuschliessen. Aus einem californischen Erz wurde durch Rösten bei Weissgluth nur wenig Osmiumsäure erhalten, während das zurückbleibende Erz in Folge des grossen Eisengehaltes zu einer krystallinischen Masse geschmolzen war.

Nach Claus ist die Methode Persoz's **), nach welcher das Osmiridium durch starkes Erhitzen bis zur Weissgluth mit einer Mischung von kohlensaurem Natron und Schwefel in die Sulfide übergeführt wird, worauf man mit Wasser die alkalischen Schwefelverbindungen auslaugt und die rückständige Masse mit Quecksilberoxyd erhitzt, wobei Iridiumoxyd zurïckbleibt, während Osmium und Quecksilber entweichen, die am wenigsten zu empfehlende Methode, weil diese Agentien das Erz nur wenig stark angreifen und die Umwandlung der Metalle in Sulfide ihre Trennung sehr complicirt. Gibbs wendete die Methode für californisches Erz an und spricht sich nicht so absolut ungünstig darüber aus. Er fand, dass die nach der Schmelzung mit Chlorwasserstoffsäure behandelten Schwefelmetalle von kochendem Königswasser und Chlorgas bei Rothglühhitze nicht merklich, dagegen durch Schmelzen mit Kali und Salpeter sehr kräftig angegriffen wurden. Da bei dieser Oxydation ein starkes Schäumen eintritt, so kann dieselbe nur in kleinem Maassstabe ausgeführt werden. Von Genth wurde dem Verf. ein Verfahren, die Schwefelmetalle vor der Oxydation

*) Journ. f. prakt. Chem. 62.340 .

**) Ebendaselbst 2. 473 . 
zu Metallen zu reduciren, mitgetheilt, welches bei sorgsamer Leitung des Processes gute Resultate gibt, jedoch mit Unbequemlichkeiten behaftet ist. Die Schwefelmetalle werden zu diesem Zwecke mit einem Ueberschuss von concentrirter Schwefelsäure zur Trockne verdampft und dann schwach geglüht. Es resultirt hierbei ein grauer, metallischer, schwammiger Rückstand, welcher nach der Methode von Claus leicht oxydirt werden kann. Die Unbequemlichkeiten, welche Gibbs dieser Methode zur Last legt, bestehen in dem Umstande, dass die Behandlung mit Schwefelsäure zwei Mal wiederbolt werden muss, und dass bei der Einwirkung der oxydirenden Agentien auf die feinzertheilten Metalle durch Spritzen leicht Verluste entstehen können. Durch allmähliches Eintragen der Metalle in kleinen Portionen in das ruhig fliessende Gemenge von Kali und Salpeter und rubiges Abwarten bis die Einwirkung vollendet ist, kann man jedoch diese Gefahr umgehen.

Wöhler's*) Methode würde nach CI aus die vorzüglichste sein, wenn nicht das vorher zu unternehmende Pulvern der Erze eine penible, zeitraubende Arbeit wäre. Wöhler's Methode besteht darin, dass man über die zur Rothgluth erhitzte Mischang von Osmiridium mit Kochsalz feuchtes Chlorgas leitet. Für pulverförmige Rückstände, besonders wenn es sich am die Gewinnnng von Rhodium handelt, ist dieselbe unstreitig die beste und empfehlenswertheste Methode. Hiermit stimmt auch Gibbs überein, indem er bemerkt, die fragliche Methode übertreffe alle andern an Eleganz, sie gebe bei Arbeiten in kleinem Maassstabe recht gute Resultate, wenn auch mehrere Wiederholungen des Prozesses erforderlich seien, um die völlige Ueberführung des Erzes in lösliche Form zu erzielen. Verf. glaubt, dass sich die Methode auch vortheilhaft für die Verarbeitung grösserer Mengen Erz eignen würde, wenn man sich grosser Porzellangefässe von plattgedrückt ellipsoidischer Form bedienen könnte, die an jedem Ende mit einer weiten Röhre von einigen Zoll Länge versehen sind.

Die neueste Methode der Aufschliessung stammt von SainteClaire-Deville und Debray; sie besteht darin, das Osmiridium mit Baryumhyperoxyd oder mit salpetersaurem Baryt aufzuschliessen. Die Anwenduug des Barytes suchen Deville und Debray mit dem Grundsatz zu motiviren: man müsse bei guten Analysen nur solche Agentien anwenden, welche durch ihre Flüchtigkeit oder die Neigung schwerlösliche Verbindungen zu bilden, aus dem Untersuchungsobject

*) Pogg. Ann. 31. 161. 
entfernt werden könnten, damit dem analysirten Körper nicht durch die Methode selbst etwas Fremdartiges mitgetheilt werde.

Claus spricht sich nun aus mehreren Gründen vollkommen ungünstig über diese Methode aus; er fübrt an, dass die Anwendung des Barytes das ganze Verfahren unnöthig complicire, ferner ist er der Ansicht, dass bei guten Analysen der Baryt gar nicht in Anwendung kommen dürfe, weil man durch das Niederreissen von Platinverbindungen durch den schwefelsauren Baryt leicht Verluste erleide. Den Grund dieses Niederfallens von Platinverbindungen mit schwefelsaurem Baryt findet Claus in der grossen Neigung der Platinmetalle, nicht nur in Form von Chlorverbindungen, sondern auch als Sauerstoffsalze schwerlösliche Doppelsalze zu bilden; so erhielt er aus Lösungen von Platinmetallen schwefelsauren Baryt, welcher so reich an Platinverbindungen war, dass er durch dieselben gefärbt erschien.

Nach dem Vorhergehenden wird man der Methode Claus' vor Allen andern den Vorzug geben müssen. Soweit es sich um die blosse Aufschliessung der Erze handelt, stimmt auch Gibbs hiermit überein, da seine Methode erst bei der Trennung der aufgeschlossenen Erze von der Claus'schen Methode abweicht.

2. Verhalten der Platinerz-Metalle zu Reagentien.

a. Verbalten des Osmiums zu Reagentien. Eine Lösung von Kalium-Osmiumsesquichlorür hat eine prachtvoll tief kirschrothe Farbe und verhält sich in folgender Weise zu Reagentien:

1) Aetzkali bewirkt sogleich einen brannröthlichen Niederschlag von Sesquioxydulhydrat, welcher zum Theil in Kali löslich ist. Beim Kochen fällt der grösste Theil dunkler gefärbt heraus. (Claus.)

2) Aetzammoniak verhält sich ähnlich. Es bildet einen Niederschlag von ammoniakhaltigem Hydrat, welches sich nach $\mathrm{Claus,} \mathrm{unter}$ wahrscheinlicher Bildung einer copulirten Base, im Ueberschuss ron Ammoniak löst.

3) Kohlensaures Kali wirkt wie Aetzkali. (Claus.)

4). Durch salpetersaures Silberoxyd wird alles Osmium in Form eines schmutzig graubrannen, in Ammoniak löslichen Niederschlages ausger̈ällt. (Cla us.)

5) Gerbsäure reducirt die Lösung unter Blaufärbung zu Chlorür (Cl.).

6) Bei längerem Erhitzen mit Weingeist unter Zusatz von etwas Salzsäure färbt sìch die Lösung violettblau (Reduction). (Claus.)

7) Schwefelwasserstoff fällt ein braunschwarzes Sulfuret, wahrscheinlich $\mathrm{Os}_{2} \mathrm{~S}_{3}$. (Cla u s.) 
8) Schwefelammonium fällt ebenfalls im Ueberschuss unlösliches Sulfuret. (Cl.)

9) Osmium in Form von Osminmsäure wird durch salpetrigsaures Kali ungemein leicht $\mathrm{zu}$ osmigsaurem Kali reducirt, welches sich in granatrothen Krystallen abscheidet. Da die Lösung sich ohne Zersetzung eindampfen lässt, so vermeidet man auf diesem Wege am besten Verluste, welche bei Anwendung anderer Methoden leicht aus der Flüchtigkeit der Osmiumsäure entstehen. In Berührung mit Papier, Staub oder andern organischen Körpern erleidet das osmigsaure Kali eine Zersetzung. unter Abscheidung von braunem Sesquioxyd. Das salpetrigsaure Kali übt auf Kalium-Osmiumchlorid keine merkliche Einwirkung aus. (Gib bs.)

b. Verhalten des Rutheniums zu Reagentien.

1) Sowohl das freie Sesquichlorid, als die Verbindung mit Chlorkalium oder Salmiak nimmt durch salpetrigsaures Kali eine orangegelbe Farbe an, ohne einen Niederschlag zu bilden. Ruthenchlorid verhält sich ebenso, wenn auch die Reaction langsamer ist und meist erst beim Erhitzen eintritt. Es bildet sich ein in Alkohol und Wasser leicht lösliches Doppelsalz, welches durch einen andauernden Strom von Schwefelwasserstoff gefällt werden kann; auch Schwefelammonium bewirkt einen Niederschlag, welcher aber durch einen Ueberschuss des Fällungsmittels wieder gelöst wird. Wenn man zu einer mit kohlensaurem Kali alkalisch gemachten Lösung einige Tropfen farbloses Schwefelammonium setzt, so entsteht eine prächtige Carmoisinfarbe, welche von keinem anderen Metalle der Platingruppe hervorgebracht wird. Ist die Menge des Ruthens gering, oder ist ein grosser Ueberschuss der anderen Metalle vorhanden, so perdampft man zur Trockne, zieht mit wenig absolutem Alkohol aus und prüft das Filtrat direct mit Schwefelammonium. (Gibbs.)

Carey Lea *) konnte die eben beschriebene Reaction mit Schwefelammonium nicht erhalten.

2) Barytwasser gibt mit Ruthenium-Ammon-Sesquichlorür in der Kälte sogleich einen Niederschlag, Ruthen-Salmiak dagegen gibt erst beim Erhitzen eine Braunfärbung und Trübung, welche jedoch durch neuen Zusatz von Barytwasser wieder gelöst wird. (Car. Lea.)

Als sehr charakteristisch für Ruthensesquichlorür beschreibt $\mathrm{Car}$. L e a folgende Reaction: Zu einer mit Ammoniak versetzten Lösung

*) Journ. f. prakt. Chem. 95. 354 . 
von unterschwefligsaurem Natron setzt man einige Tropfen Ruthensesquichlorür. Die Lösung nimmt hierdureh eine so intensiv purpurrothe Farbe an, dass man mit Hülfe dieser Reaction noch 1/100000 des Ruthensalzes erkennen kann. Gegenwart von Iridium hindert diese Reaction nicht und der Verf. empfiehlt dieselbe daher, um in diesem die kleinste Beimengung von Ruthenium zu finden. Car. Lea führt ubbrigens an, dass die Empfindlichleit dieser Reaction doch gewisse Grenzen habe, so sei bei einem Verhältnisse von 1 Thl. Ruthensesquichlorid auf 500 Thle. Iridiumsalmiak die hervorgebrachte Färbung nicht mehr rosa, sondern orange. Mittelst der belannten Reactionen von Schwefelcyankalium and Bleizucker kamn man Ruthen schon bei einem Verhältnisse von $1 \mathrm{Thl}$. Ruthensalz auf 50 Thle. Iridiumsalz nicht mekr erkennen. Wie Cla as sebon beobachtet hat, zersetzen sich die Lösungen von Ruthensesquichlorür in verdünntem Zustande leicht unter Abscheidung von Ruthensesquioxydul; will man sich daher vor der Gefahr geringe Ruthenmengen zu übersehen, schützen, so muss man die zu prüfende Lösung kurz vor der Anwendung mit etwas Salzsäure kochen, mit 'Ammon übersättigen und sogleich' das unterschwefiigsaure Natron zusetzen. (Car. Lea.)

3) Tetrathionsäure fällt Ruthenchlorid beim Kochen in saurer Lösung braun, während ammoniakalische Lösung gelb gefärbt wird. Ruthensesquichlorid wird beim Kochen entfärbt und später gran getrubt. (Car. Lea.)

4) Verdünnte Lõsungen von Ruthensesquichlorid werden beim Kochen mit Zinnchlorür farblos, concentrirte gelblich. Unterschied von Iridiumsesquichlorür. (Car. Lea.)

5) $\mathrm{Zu}$ wässerigem Ammon setzt man so lange Chlorzink bis der Geruch des ersteren beinahe verschwunden und etwas Zinkoxyd angelöst geblieben ist. Eine Lösung von Ruthenchlorid wird durch diese Nischung rosenroth, eine Lösung von Ruthensesquichlorid brain gefällt. Der Niederschlag ist Zinkoxyd, welches durch mit niedergerissenes Salz gefärbt erscheint. (Car. L e a.)

6) Kaliumeisencyanid in Natronlauge gelöst färbt Ruthensesquichlorür hellgelb, Ruthenchlorid mehr weingelb. (Car. Lea.)

7) Vermischt man eine Lösung des Schlippe'schen Salzes mit gleichem Volum Ammon, so erzeugt diese Lösung mit Ruthensesquichlorür beim Kochen einen schwärzlichen Niedersehlag, während eine kochende Lösung von Rathenchlorid mit einem Tropfen des Reagens eine gelbe durchsichtige Flüssigkeit, mit einer grösseren Menge des- 
selben dagegen schon bei gelindem Erwärmen einen ziegelfarbigen Nieschlag erzeugt. (Car. Lea.)

c. Verhalten des Iridiums zu Reagentien.

1) Kalium oder Ammonium-Iridiumchlorid wird durch salpetersaures Kali sogleich olivengrün gefärbt; nimmt man die Reaction in heisser Lösung vor, so krystallisirt beim Erkalten Iridium-Kalium- oder IridiumAmmoniumsesquichlorid aus. $2\left(\mathrm{KCl}+\mathrm{IrCl}_{2}\right)+\mathrm{KO}, \mathrm{NO}_{3}=3 \mathrm{KCl}$, $\mathrm{Ir}_{2} \mathrm{Cl}_{3}+\mathrm{NO}_{4}$. Wenn man aber einen Ueberschuss von salpetrigsaurem Kali anwendet, die Lösung kocht oder freiwillig verdunsten lässt, so geht die grüne Farbe in eine gelbe über und es scheidet sich bei weiterem Kochen ein Theil des Iridiums als ein schweres, schweeweisses Pulver aus; das Pulver ist in kaltem Wasser unlöslich, in kochendem nur spärlich löslich und wird von kochender Salzsäure fast gar nicht angegriffen. Durch Einwirkung von salpetrigsaurem Natron erhält man ein gelbes Doppelsalz, welches selbst beim Kochen mit Schwefelalkalien keinen Niederschlag gibt. (Gibbs.)

2) Barytwasser lösst den Iridiumsalmiak schnell zu einer farblosen Flüssigkeit and gleichzeitig bildet sich ein olivengrüner Niederschlag. Erwärmt man das Filtrat von diesem Niederschlag, so färbt es sich dunkel olivengrün und dann plötzlich isabellfarbig, während ein dicker, blass graugelber oder gelblichbrauner Niederschlag sich ausscheidet. Der olivengrüne Niederschlag löst sich in Säuren mit olivengrüner Farbe unter Hinterlassung eines schweren schwarzen Pulvers; der gelbbraune löst sich frisch bereitet ebenfalls mit olivengrüner, bei $100^{\circ}$. getrocknet aber mit blauer Farbe. Dieselbe Farbe nimmt er anch an, wenn er trocken mit Kalilauge übergossen wird. (Car. Lea.)

3) Durch eine mit Ammon versetzte Lösung von unterschwefligsaurem Natron wird Iridiumsalmiak entfärbt. (Ca r. L ea.)

4) Iridchlorid wird beim Kochen mit Tetrathionsäure entfärbt, Iridsesquichlorür wird in saurer Lösung durch das gleiche Reagens weinroth gefärbt, in ammoniakalischer jedoch nicht verändert. (Car. L ea.)

5) Mit Zinnchlorür gibt Iridsesquichlorür einen Niederchlag, der sich in Kali löst und sich bei nachherigem Kochen mit Lederfarbe wieder ausscheidet. Unterschied von Ruthensesquichlorür. (C a r. Lea.)

6) Eine ammoniakalische Lösung von Zinnchlorid gibt mit Iridsesquichlorürlösungen einen blass ledergelben, mit Iridchloridlösungen einen feuerrothen Niederschlag. (Car. Lea.)

7) Eine Lösung von Kaliumeisencyanid in Natronlauge färbt eine ammoniakalische Iridsesquichlorürlösung glänzend gelb, im Kochen tief 
weinroth, die saure Lösung dagegen ist grun, kochend olivengrün. (Car. Lea.)

8) Iridsesquichlorür gibt mit Schlippe'schem Salz und gleichem Volum Ammon versetzt, einen ziegelfarbigen Niedersehlag. - Iridchlorid wird entfärbt and gibt beim Kochen einen ziegelbraunen Niederschlag. (Car. Lea.)

d. Verhalten des Palladiums zu Reagentien.

1) Nach F ischer bildet sowohl Palladiumchlorür als auch Palladiumchlorid mit salpetrigsaurem Kali ein in Wasser lösliches, durch Alkohol fällbares Doppelsalz.

2) Palladiumchlorür gibt mit Barytwasser einen, im Ueberschuss des Fällungsmittels unlöslichen Niederschlag. (Car. Lea.)

3) Durch eine mit Ammon versetzte Lösung von unterschwefligsaurem Natron färbt sich Palladiumchlorür kalt citronengelb, beim Kochen schnell braun. (Car. Lea.)

4) Tetrathionsäure färbt eine verdünnte Palladiumchlorürlösung in der Kälte braun, in einer weniger verdünnten bewirkt sie einen braunen Niederschlag. Unterscheidung von Ruthen, Iridium, Platin. (Car. Lea.)

5) Schwefelsaures Chinin erzeugt in Palladiumchlorür einen lederfarbigen Niederschlag. Unterschied von Ruthen und Iridium. (Car. Lea.)

6) Palladiumchlorür als Ammoniakdoppelsalz angewendet gibt mit einer Lösung von Schlippe'schem Salz mit einem gleichen Volum Ammon versetzt, einen schwarzbraunen Niederschlag.

e. Verhalten des Rhodiums zu Reagentien.

1) Rhodiumsesquichlorid wird durch salpetrigsaures 'Kali beim Erhitzen gelb. Beim Kochen oder Verdampfen scheidet sich ein Theil des Rhodiums in Form eines orangegelben, in Wasser sehr schwer, in Salzsäure leicht löslichen krystallinischen Pulvers aus. Ein anderer Theil bleibt in Lösung and kann durch Zusatz von Weingeist gefällt werden. Aus beiden Salzen fällen Schwefelalkalien im Ueberschuss lösliches, durch Salzsäure wieder vollkommen fällbares Sulfuret. (Gibbs.)

2) Barytwasser fällt Rhodiumsesquichlorür sofort als hellen, im Ueberschuss von Barytwasser leicht löslichen Niederschlag. Untersehied von Ruthenchlorid. (C a r. Lea.)

3) Rhodiumsesquichlorür wird durch eine mit Ammon versetzte Lösung von unterschwefligsaurem Natron strohgelb gefärbt. (Car. L e a.) 
f. Verhalten des Platins zu Reagentien.

1) Platin wird in Form von Kalium- oder Ammoniumdoppelsalz durch salpetrigsaures Alkali nicht wesentlich verändert. (Gibbs.)

2) Barytwasser äussert auf Platinchlorid in der Kälte kaum eine Wirkung, beim Erwärmen fällt aus der gelb bleibenden Lösung ein schmutzig weisser Bodensatz. (C ar. Le a.)

3. Durch eine mit Ammon versetzte Lösung von unterschwefligsaurem Natron wird aus Platinchlorid anfangs Platinsalmiak gefällt, dann entsteht eine gelbe, bei weiterem Kochen farblose, schliesslich tief weinrothe Lösung. (Car. L e a.)

4) Tetrathionsäure ertheilt einer Lösung ron Platinchlorid eine weinrothe Färbung. (C a r. Lea.)

5) Schlip pe'sches Salz, ohne Ammoniak angewendet, fällt aus Platinchlorid in der Kälte einen ziegelbraunen Niederschlag.

3. Trennung der Metalle der Platingruppe.

Nach $\mathrm{Cl}$ aus verfährt man also:

Die Ruthenlösung A (siebe Claus' Methode der Aufschliessung p. 118) wird mit sehr verdünnter Salpetersäure vorsichtig neutralisirt. Unter starkem Aufbrausen durch Fintweichen von Stickoxydgas fällt ein reichlicher sammtschwarzer Niedersehlag $b$, während ein starker Geruch nach Osmiumsäure diese Operation, besonders beim Arbeiten mit grossen Mengen, zu einer höchst unangenehmen macht. Wenn sich derNiederschlag b abgesetzt hat, so wird die klare Flüssigkeit a abgegossen und der Niederschlag, welcher aus Osmiumoxydhydrat $\mathrm{OsO}_{2}, 2 \mathrm{HO}$ und etwa 15-20 Proc. Ruthensesquioxydul besteht, auf einem Filter gesammelt und ansgewaschen.

Aus diesem Niederschlage kann man das schöne rothe Ruthensalz mit geringer Mühe erhalten. $\mathrm{Cl}$ a us erhielt $240 \mathrm{Grm}$. des Niederschlags $b$ und aus diesem etwas mehr als 30 Grm. Ruthenoxydulhydrat. $\mathrm{Zu}$ diesem Behufe wird derselbe, um ihn von Osmium zu trennen, in einer Retorte mit grosser Vorlage, welche wegen der giftigen Eigenschaften der leicht flüchtigen Osmiumsäure luftdicht angepasst sein muss, mit 2 Pfund Salzsäure und 3 Pfund Salpetersäure destillirt. Die Destillation, welche man am besten im Winter ausführt um mit Schnee kühlen zu können, wird langsam and vorsichtig geleitet bis der Rest in der Retorte dickflüssig geworden and die meiste Säure abdestillirt ist. Der Rückstand in der Retorte besteht dann aus $\mathrm{KuCl}_{2}$ mit geringen Antheilen $\mathrm{Ra}_{2} \mathrm{Cl}_{3}$, kann aber auch noch Spuren von Osmium enthalten. Um sich von der Abwesenheit des Osmiums 
zu überzeugen erhitzt Claus eine Probe in einem Platinlöffelchen und hält den glühenden Rest alternativ in den oxydirenden und nichtoxydirenden Theil einer Weingeistflamme, Zeigt sich ein momentanes, sehr starkes Leuchten in der Oxydationsflamme, so ist noch Osmium vorhanden und die Destillation mit Salz- und Salpetersäure muss so oft wiederholt werden, bis das Ausbleiben dieser Erscheinung die Abwesenheit allen Osmiums in der Ruthenverbindung anzeigt.

Das nun osmiumfreie Ruthenchlorid löst man in der Retorte in einer möglichst geringen Menge siedenden Wassers, fügt etwas Salmiak hinzu und lässt erkalten. War Ruthensesquichlorür in dem Chlorid, so scheidet es sich nach längerem Stehen als braunes Doppelsalz $2 \mathrm{NH}_{4} \mathrm{Cl}+\mathrm{Ru}_{2} \mathrm{Cl}_{3}$ aus, während das Chlorid mit tief kirschrother Farbe in Lösung bleibt. Nachdem man das braune Salz entfernt hat, löst man viel Salmiakpolver in der erhitzten Chloridlösung, worauf sich dann beim Erkalten das Ruthenchlorid in Verbindung mit Salmiak als tief rothes Krystallmehl abscheidet. Da die Mutterlauge noch viel Salz enthält, so wird sie rasch eingedampft, wobei sich schon in der Siedhitze das Salz grösstentheils als Krystallmehl ausscheidet. Auf einem Filter gesammelt wird das Ganze nun mit Weingeist ausgewaschen und so der Salmiak entfernt. Will man jede Spur braunes Salz entfernen, so übergiesst man mit wenig Wasser, so dass noch ein geringer Antheil ungelöst bleibt, man lässt einige Zeit stehen, giesst die sehr concentrirte Lösung $a b$ und überlässt sie der freiwilligen Verdunstung. Man erhält nun grössere octaëdrische Krystalle, welche dem rothen Rhodiumsalze $3 \mathrm{NH}_{4} \mathrm{Cl}+\mathrm{Rh}_{2} \mathrm{Cl}_{3}$ zum Verwechseln ähnlich sehen; wird es jedoch geglüht, so erhält man das schönste reinste Ruthenium in Form eines lockeren, leichten silberweissen Ruthenschwammes.

Die Flüssigkeit a, aus welcher durch Salpetersäure das Gemenge von $\mathrm{OsO}_{2}$ und $\mathrm{Ru}_{2} \mathrm{O}_{3}$ gefällt worden ist, enthält noch grössere Mengen von Ruthenoxyd, Ruthenhypersäure neben viel freier Osminmsäure. Um nun das Osmium zu trennen destillirt man die Lösung mit ein paar Pfund Salzsäure. Man setzt das Destilliren so lange fort, bis alles Osmium entwichen ist and die Lauge in der Retorte eine rothe Farbe angenommen hat. Die Lauge wird jetzt concentrirt und durch Auskrystallisirenlassen von dem grössten Theil des Salpeters befreit, darauf zur Trockene verdampft; das trockene Salz wird wieder gelöst und das Ruthen durch Schwefelammonium unter Zusatz etwas freier Sänre gefällt. Durch Glühen kann man das Schwefelruthen in Ruthenoxyd oder durch Behandeln mit Salpetersäure in schwefelsaures Ruthen- 
oxyd überführen. Aus dem ursprünglichen Ruthenauszug gewinnt man das Ruthen nach der eben beschriebenen Methode.

Zur Darstellung des Osmiums unterwirft man alle durch Destillation gewonnenen osmiumsäurehaltigen Flüssigkeiten der Destillation, wobei die Osmiumsäure viel früher übergeht als die Salpeter- und Salzsäure und man im Destillat eine concentrirte Lösung von $\mathrm{OsO}_{4}$ mit geringen Mengen der anderen Säuren verunreinigt erhält. Diese Lösung kanu man entweder direct mit Schwefelwasserstoff fällen oder die Osmiumsäure durch Aetzkali und Alkohol in osmigsaures Kali überführen dann Salmiakpulver hinzuthun, wobei der grösste Theil des Osmiums als isabellgelbes Osmamid-Chlorammonium (die Chlorverbindung der Osmiumbase) ausgefüllt wird. (Frem y.)

Claus bemerkt noch, dass man bei dem eben beschriebenen Schmelzverfahren nicht zu fürchten hat, in die alkalische Ruthenlösung auch Iridium übergehen zu sehen. Ruthen and osmiumreiche Erze theilen nämlich dem Schmelzproducte kein basisch-iridiumsaures Kali mit, während ruthen- und osmiumarme Erze, namentlich die körnigen, der Schmelze so viel Iridium als iridiumsaures Kali mittheilen können, dass dieselbe eine grünliche, ja sogar tiefblaue Lösung gibt. Aus diesem Grunde erscheint es anch nicht räthlich, dem schwarzen Pulver B durch öfter wiederholte Schmelzungen alles Ruthen and Osmium entziehen zu wollen; das durch die ersten Schmelzungen schon sehr ruthenund osmiumarme Pulver würde hierbei leicht Iridium an die Schmelze abgeben. Man gewinnt daher aus diesem Pulver das Ruthen und Iridium auf folgende Weise. Man unterwirft $\mathbf{B}$ mit einem Ueberschuss von Königswasser der Destillation, wobei das Osmium als Osmiumsäure übergeht, und die anderen Metalle bis auf die unlöslichen Verbindungen von Rhodium, Platin, etwas Iridiumoxyd, und einen geringen Rest unaufgeschlossenen Erzes sich auflösen. Sobald die Lösung nicht mehr nach Osmiumsäure riecht, wird sie in Porzellanschalen stark eingeengt und einige Zeit zum Erkalten hingestellt. Das meiste Iridium scheidet sich als schwarzes Krystallpulver von Kalium-Iridiumchlorid aus. Die concentrirte Mutterlange wird von dem Iridiumsalz durch Filtriren getrennt und etwas Salmiak zugesetzt, wodureh der letzte Rest des Iridiums als Ammonium-Iridiumchlorid ausgeschieden wird, während das Ruthen in Lösung bleibt. Es wird filtrirt, nachdem man längere Zeit hat stehen lassen und nun dieser letzten Mutterlauge viel Salmiakpulver zugesetzt. Nach einiger Zeit gesteht die Masse zu einem braunen Brei von Krystallmehl, welches auf einem Filter gesammelt, erst mit Salmiaklösung, dann mit Weingeist ausgewaschen, fast reines braunes 
Ruthensalz zurtucklässt. $\mathrm{Cl}$ a us gewann auf diese Weise noch $30 \mathrm{Grm}$. $2 \mathrm{NH}_{4} \mathrm{Cl}+\mathrm{Ru}_{2} \mathrm{Cl}_{3}$ und $450 \mathrm{Grm} . \mathrm{KCl}, \mathrm{IrCl}_{2}$. Dieses Iridiumsalz entbält noch etwas Ruthen, welches durch fractionirte Lösung davon getrennt werden kann und etwas Platin.

$\mathrm{Cla}$ as bespricht schliesslich noch die Gewinnung des Rhodiums aus den rhodiumreichen Rückständen, nämlich dem schwarzen Metallpulver, welches aus den Mutterlaugen der bearbeiteten Platinerzlösungen durch Fällen mit Eisen gewonnen wird. Nach Deville and Debray werden diese Rückstände durch Schmelzen mit Blei und Bleiglätte gereinigt. Die Platinmetalle legiren sich mit dem Blei, welches durch verdünnte Salpetersänre ausgezogen werden kann, während die Unreinigkeiten, d. h. die anderen beigemischten Mineralkörper, in die Bleischlacken gehen. Diese Reinigungsmethode empfiehlt $\mathrm{Cl}$ aus angelegentlich; indem er sie für weit besser als die von ihm selbst vorgeschlagene erklärt; dagegen hält er die Aufschliessung mit Baryumhyperoxyd oder salpetersaurem Baryt für anpraktisch und im Princip verfehlt, weil das auf diese Weise entstehende Rhodiumoxyd in Säuren vollkommen unlöslich sei. Claus empfiehlt hier die oben schon beschriebene Methode Wöhler's. Das Rhodium in feinzertheiltem Zustande wird nämlich von allen Platinmetallen am leichtesten angegriffen; aus einer Mischung von viel Iridium mit wenig Rhodium erhält man daher bei Anwendung dieser Methode eine an Rhodium reichere Lösung, als den gegenseitigen Mengenverhältnissen beider Metalle entspricht; so kann ein Rückstand, welcher 10 Proc. Rhodium enthält, eine 30procentige Lösung geben. Dieselben Rückstände, welche, mit SchmelzmitteIn behandelt, keine Spur Rhodium erkennen lassen, enthalten häufig geringe Mengên dieses Metalles, welche sich nach Wöhler's Methode leicht gewinnen lassen. Bei Ausführung der Wöhler'schen Methode wendet man am besten grössere Porzellanröhren an und lässt einen starken Strom feuchten Chlorgases bei nicht zu starker Hitze einwirken. Schmilzt das Gemenge von Metallpulver und Kochsalz zu Anfang der Operation, so senkt sich das Metall za Boden und wird so der Einwirkung des Chlors entzogen. Die gewonnenen Lösungen muss man mit etwas Salpetersäure erhitzen, um das Iridiumsesquichlorür in Chlorid zu verwandeln, da das mit dem Rhodiumsalz isomorphe Sesquichlorür sich nicht pon demselben trennen lässt. Durch fractionirte Fällung mit Salmiakpulver wird endlich das Iridiumsalz von dem Rhodiumsalze getrennt. $\mathrm{Claus}$ betont die fractionirte $\mathrm{F}$ ällung, weil Deville und Debray durch Lösung des zur Trockne eingedampften Salzgemenges in Salmiaklauge, beide Metalle zu trennen 
versuchen. Hat sich aber nach $\mathrm{Claus}$ einmal das Rhodiumsalz ausgeschieden, so wird es in Salmiakwasser bedeutend unlöslicher und haftet auch dem Iridiumsalze stärker an, welcher Umstand die Anwendung einer grossen Salmiakmenge nöthig macht.

Gegen diese Methode wendet $\mathrm{Gibbs} *$ ) ein, dass die Trennung des Osmiums und Rutheniums durch die grosse Menge der, zur Lösung der auflöslichen Theile erforderlichen Flüssigkeit, und die daraus folgende Nothwendigkeit sehr grosse Retorten anwenden zu müssen, unbequem werde und dass man sich ferner nicht genügend vor den Dämpfen der Osmiumsäure schützen könne. Gibbs bringt daher folgende Methode in Vorschlag:

Man kocht die geschmolzene, zerkleinerte Masse in einem eisernen Gefässe so lange mit Wasser, dem ein Zehntel seines Volums Weingeist zugesetzt wird, bis die Masse vollkommen zertheilt erscheint. Bei diesem Process wird das osmiumsaure Kali zu osmigsaurem Kali reducirt, das ruthensaure Kali wird gänzlich zerlegt und das Ruthenium als schwarzes Oxydgemenge abgeschieden. Nachdem man die Flüssigkeit mit dem leichten Bodensatze abgegossen hat, wird der Rückstand nochmals mit Alkohol and Wasser gekocht und nach einigem Stehen abgegossen.

Durch diese Behandlungsweise erhält man 1) eine Lösung von osmigsaurem Kali; 2) eine grosse Menge schwarzer Oxyde und 3) einen Rest unzersetzten Erzes in Form eines schweren groben Pulvers. Dieses letztere wird nun wieder mit Kali und Salpeter aufgeschlossen und nach der eben angegebenen Methode weiter behandelt. Die klare Lösnng des osmigsauren Kalis wird nun mit einem Heber abgezogen und die schwarzen Oxyde, welche noch einen Theil des im Erze enthaltenen Osmiums zurückhalten, mit heissem Wasser und Alkohol ausgewaschen, darauf in einer geräumigen tubulirten Retorte durch einen Sicherheitstrichter allmählich mit starker Chlorwasserstoffsäure übergossen. Durch sehr heftige Reaction, welche hierbei eintritt, wird so viel Hitze entbunden, dass ein Theil der Osmiumsäure ohne weiteres Erwärmen überdestillirt. Die sehr dicht angepasste Vorlage wird mittelst weiter Abzugsröhren ( um ein Verstopfen derselben zu verhüten) mit 2 oder 3 doppelhalsigen Flaschen, welche eine starke Kalilösung und etwas Alkohol enthalten, verbunden und, nachdem die Einwirkung in der Retorte aufgehört hat, wird die Destillation durch Erhitzen auf

*) Americ. Journ. of Sciences and Arts. 2 Ser. Vol. XXXI; Journ. f. prakt. Chem. 84. 68. 
dem Sandbade so lange fortgesetzt, bis die im Retortenhals condensirte Osmiumsäure in Tropfen in die Vorlage abgeflossen ist. Sobald die Retorte abgekühlt ist, nimmt man die Vorlage ab und verschliesst sie fest mit einem Kork; hierauf treibt man die condensirte Osmiumsäure durch Erhitzen der Vorlage im Wasserbad in die doppelhalsigen Flaschen, vereinigt ihren Inhalt mit der direct durch Schmelzen erhaltenen Lösung von osmigsaurem Kali, verdampft und stellt zum Krystallisiren an einen kühlen Ort. Auf diesem Wege erbält man das osmigsaure Kali in Krystallen, welche in Salzlösungen so schwerlöslich sind, dass man die Mutterlange als werthlos wegwerfen kann.

Die in der Retorte zurückbleibende Flüssigkeit, welche Eisen, Palladium, Platin, lridium, Rhodium und Ruthenium nebst Spuren von Osmiumsäure enthält, wird, um diese letzere zu entfernen, so lange, mit Wasser und Salzsäure versetzt, eingedampft bis sich kein Geruch nach Osmiumsäure mehr bemerklich macht. Man setzt hierauf eine kalte, gesättigte Chlorkalinmlösung zu, wodurch Eisenchlorid und Palladiumchlorid gelöst werden, während die Chloride von Platin, Iridium, Rhodium und Ruthenium in Form unlöslicher Doppelsalze zurückbleiben. Gibbs führt nicht an, auf welche Weise er die Trennung dieser $\mathrm{Me}$ talle bewerkstelligt hat, sondern begnügt sich mit der Angabe, dass die Doppelsalze zuerst mit einer gesättigten Clorkalinmlösung, daranf mit einer Salmiaklösung ausgewaschen werden.

In einer späteren Abhandlung*) beschreibt der Verf. die Trennung der ansgewaschenen Doppelchloride auf Grund ihres verschiedenen Verhaltens zu salpetrigsauren Alkalien und ihrer verschiedenen Löslichkeit folgendermaassen.

Platin von Iridium. Die Kaliumdoppelchloride $\mathrm{KCl}_{2} \mathrm{PtCl}_{2}$ and $\mathrm{KCl}_{2}, \mathrm{IrCl}_{2}$ werden fein zerrieben, mit 3 Volum kochendem Wasser übergossen und vorsichtig so lange verdünnte Lösung von salpetrigsaurem Kali zugesetzt, bis die Lösung tief grün erscheint; die Flüssigkeit wird durch Zusatz von kolllensaurem Kali neutral erhalten. Die grüne Lösung wird abgegossen und die Behandlung mit salpetrigsaurem Kali fortgesetzt bis sich die Lösung nicht mehr grün färbt. Aus der grünen Lösung erhält man durch Abdampfen Krystalle von $3 \mathrm{KCl}+\mathrm{Ir}_{2} \mathrm{Cl}_{3}+6 \mathrm{HO}$, welche sich durch Umkrystallisiren von der letzten Spur Platin befreien lassen. Die Anwendung von salpetrigsaurem Natron hat den Vortheil, dass, im Falle ein Ueberschuss von Nitrit angewendet wurde, das ent-

*) Americ. Journ. of Science and Arts 34, p. 341; Journ. f. prakt. Chem. 91. 191. 
stehende Doppelsalz durch Salzsảure leicht in $\mathrm{NaCl}, \mathrm{IrCl}_{2}$ übergeführt werden kann; durch vorsichtigen Zusatz einer verdünnten Lösung von salpetrigsaurem Natron zu der mit kohlensaurem Natron neutralisirten Lösung kann man dann leicht das Doppelsalz $3 \mathrm{NaCl}+\mathrm{Ir}_{2} \mathrm{Cl}_{3}+24 \mathrm{HO}$ wieder herstellen. Das Iridiumsalz ist vollkommen platinfrei, während dagegen dem Ruckstand noch etwas Iridium anhaftet. Sind ausser Platin noch andere Metalle zugegen, so erleidet die Methode eine Modification: Man verfährt wie vorhin um den grössten Theil des Platins abzuscheiden und kocht die filtrirte Lösung mit einem Ueberschuss von salpetrigsaurem Natron bis sie gelb geworden ist. Hierauf wird vorsichtig Schwefelnatrium zugesetzt bis der entstandene Niederschlag von Schwefelplatin sich theilweise wieder gelöst hat, und mit Salzsäure schwach angesäuert. Dss Platinsulfid wird hierdurch gefällt, es wird mit heissem Wasser ausgewasehen und durch Kochen mit Salzsäure das $3 \mathrm{NaCl}+\mathrm{Ir}_{2} \mathrm{Cl}_{3}$ in $\mathrm{NaClICl}_{2}$ übergefübrt. Die Lösung desselben wird verdampft, mit starker Salmiaklösung gefällt und ausgewaschen. Der Iridiumsalmiak, der anf diesem Wege gewonnen wird, liefert chemisch reines Iridium.

Platinvon Ruthenium. Während eine oberfiächliche Trennung beider Metalle einfach durch Behandeln ibrer Doppelchloride mit einer concentrirten, kalten Lösung von Chlorkalium erreicht werden kam, wobej jedoch in die Lösung des Ruthensalzes immer Platinspuren übergehen und in dem zurückbleibenden Platinsalz stets noch Ruthen enthalten ist, erfordert die rollständige Trennung beider Metalle ein ziemlich weitläufiges Verfahren. Gibbs verwandelt die nach soeben angegebener Methode erhaltene Lösung von Kalium-Ruthenchlorid durch Kochen mit salpetrigsaurem Kali in das gelbe Doppelsalz, verdampft im Wasserbade zar Trockne and kocht aus dem Rückstand das leichtlösliche Ruthensalz mit Alkohol aus. Diese Lösung enthält noch etwas Platin; sie wird zur Trockne verdampit, der Rückstand mit Salzsäure behandelt, die rothe Lösung des Kalium-Ruthenchlorides mit einem Ueberschuss von Salmiak zur Trockne verdampft, nochmals gelöst und verdampft, and endlich die trockene Masse mit kaltem Wasser behandelt. Im Rückstand hat man ein Gemisch von $\mathrm{KCl}, \mathrm{RuCl}_{2}$ und $\mathrm{NH}_{4} \mathrm{Cl}$, $\mathrm{RuCl}_{2}$, welches in heissem Wasser gelöst und mit Ammoniak bis zur Gelbfärbung gekocht wird. Zur Trockne verdampft bleibt $2 \mathrm{NH}_{3}, \mathrm{RuCl}$ + 3HO; in Wasser gelöst gibt diess mit Sublimat einen schönen gelben, in kaltem Wasser schwer, in heissem leicht löslichen krystallinischen Niederschlag von $2 \mathrm{NH}_{3}, \mathrm{RuCl}+\mathrm{HgCl}$, welchen man durch Umkarystallisiren leicht von Platin frei erhalten kann. Beim Glühen hinterlässt 
derselbe reines silberweisses Ruthenium. Wenn Ruthensesquichlorür vorhanden ist, so muss dieses durch Behandeln mit salpetrigsaurem und kohlensaurem $\mathrm{Kali}$, wie oben angegeben, in $\mathrm{RuCl}_{2}, \mathrm{KCl}$ übergeführt werden.

Iridium von Ruthenium. Die Lösung beider Metalle wird mit salpetrigsaurem Natron und kohlensaurem Natron gekocht bis die Lösung orangefarben erscheint, man fügt darauf in kleinen Mengen Schwefelnatrium zu bis zur Lösung eines Theils des gefällten Ruthens, kocht kurze Zeit und fügt nach vollständigem Erkalten vorsichtig Salzsäure bis zur schwach sauren Reaction hinzu. Das gefällte Schwefelruthen wird mit heissem Wasser ausgewaschen und im Filtrat, welches kein Ruthen mehr enthält, das Iridium durch Behandeln mit Salzsäure und Salmiak in Iridium-Ammoniumchlorid übergeführt. Durch Erhitzen des ausgewaschenen Schwefelruthens sammt dem Filter mit concentrirter Salzsäure, Salmiak und allmählich zugesetzter Salpetersäure wird dasselbe gelöst, die Lösung znr Trockene verdampft und durch Auswaschen mit concentrirter Salmiaklösung das $\mathrm{NH}_{4} \mathrm{Cl}, \mathrm{RuCl}_{2}$ ii reinster Form erhalten. Dieses wird, wie oben gezeigt, in $2 \mathrm{NH}_{3}$, $\mathrm{RaCl}+\mathrm{HgCl}$ übergeführt und aus dieser Verbindnng durch Glühen im Wasserstoffstrom reines Rathen gewonnen.

Der Verfasser beschreibt noch folgende Trennung des Iridiumchlorids vom Ruthenchlorid, welche so genaue Resultate liefert, dass er dieselbe zu quantitativen Bestimmungen empfiehlt. Die Lösung beider Chloride wird mit überschüssigem salpetrigsaurem and kohlensaurem Kali gekocht, zur Trockene verdampft und das RutheniumKali-Doppelnitrit mit absolutem Alkohol ausgezogen. Das Kali-IridiumDoppelnitrit bleibt unlöslich zurück and wird mit Salmiaklösung in Iridiumsalmiak verwandelt.

Durch Schmelzen mit Kali and Salpeter lässt sich eine Trennung des Iridiums rom Ruthenium nicht erreichen, weil mit dem ruthensauren Kali immer auch Iridium in Lösung geht.

Iridium von Rhodium. Die Trennung kleiner Rhodiummengen von Iridium, für welche sich die Clans'sche Methode als unzulänglich erwiesen hat (Gibbs), gelingt nach demselben Verfahren, welches für die Trennung des Iridiums rom Ruthenium empfohlen wurde. Das Schwefelrbodium verwandelt man auf dieselbe Art wie das Schwefelruthen in Ammonium-Rhodiumsesquichlorür, wäscht mit einer kalten, gesättigten Salmiaklösung aus und verwandelt das umkrystallisirte Salz schliesslich in $5 \mathrm{NH}_{3}, \mathrm{Rh}_{2} \mathrm{Cl}_{3}$, welches frei von Iridium erhalten wird. 
Rhodium von. Ruthenium. Man behandelt wie bei der Trennung des Platins vom Ruthen mit salpetrigsaurem Kali und extrahirt mittelst Alkohols das Ruthensalz; ungelöst bleibt das Doppelnitrit von Kali und Rhodium zurück. Dieses kann man nun entweder mit einem Ueberschuss von Salmiak glühen, wobei metallisches Rhodium mit Chlorkalium zurückbleibt, oder man kann in heisser Salzsäure lösen und in ammoniakalischer Lösung mit Schwefelalkalimetall das Rhodium als Schwefelrhodium fällen, welches wie oben weiter behandelt wird. Die Behandlung mit salpetrigsaurem Kali kann man, um die letzten Ruthenspuren $z u$ entfernen, zweimal wiederholen.

$0 \mathrm{smium} \mathrm{kann} \mathrm{man} \mathrm{durch} \mathrm{Verflüchtigen} \mathrm{leicht} \mathrm{von} \mathrm{allen} \mathrm{übrigen}$ Platinmetallen trennen.

Palladium wird am besten durch das gewöbnliche, auf der Leichtlöslichkeit seiner Alkalidoppelsalze in Chloralkalimetalllösungen beruhende, Verfahren getrennt.

Die Trennungämmtlicher Metalle der Platingruppe mit Ausnahmedes Osminms und Palladiums. Auf Grundlage des beschriebenen Verhaltens der Metalle der Platingruppe schlägt Gibbs folgende Trennungsmethode vor: Die Chloride werden mit ihrem 4- bis 5 -fachen Volumen kochenden Wassers in einer tiefen Porzellanschale übergossen und mit dem halben Gewichte salpetrigsanrem und etwas kohlensaurem (bis zu alkalischer Reaction) Natron durchgerührt. Diese Operation wird wiederholt, so lange das Filtrat sich noch grün färbt. Der Rückstand besteht dann im Wesentlichen aus Eisenoxyd und Erzbeimengungen, während im Filtrat die Sesquichlorüre des Iridiums und Rhodiums, Chlorid and Chlorür des Rutheniums, endlich Platinchlorid enthalten ist. Das letztere scheidet sich beim Erkalten zum grössten Theil mit etwas Iridiumkaliumehlorid in Krystallen aus.

Das Filtrat kocht man mit salpetrigsaurem Natron bis es eine helle Orangefarbe angenommen hat, fällt mit Schwefelnatrium und sänert schliesslich mit Salzsäure schwach an. Man filtrirt und behandelt das Filtrat noch einmal nach der angegebenen Methode, wobei noch ein kleiner Theil Sulfide erhalten wird. Aus diesem zweiten Filtrat, welches nur noch Iridium enthält, gewinnt man das letztere nach einer beliebigen Methode.

Die ansgewaschenen Schwefelmetalle werden nun in Königswasser gelöst, die Lösung zur Trockene verdampft und daranf mit Wasser aufgenommen. Um die Sulfate zu beseitigen, fällt man durch Zink und behandelt die fein zertheilten Metalle nach dem Auswaschen und 
Trockenen, nachdem man sie mit Chlorkalium gemischt hat, in niederer Rothgluth mit Chlorgas. Mittelst salpetrigsauren Kalis werden die erhaltenen Doppelchloride in Doppelnitrite rerwandelt. Diese zieht man mit Alkohol aus, welcher alles Ruthensalz löst und die Doppelnitrite des Platins und Rhodiums zurücklässt. Das Ruthensalz kann man dann, wie oben gezeigt, in die Ammoniumbasis überführen und überhaupt nach der beschriebenen Methode reines Ruthen darans erhalten. Durch Behandeln der Doppelnitrite mit Salzsäure, mehrmaliges Auflösen in Wasser mit darauffolgendem Eindampfen zur Trockene, führt man das Rhodium in unlöslichen Zustand über und trennt hieranf Platin und Rhodium durch kochendes Wasser, welches Platinkaliumchlorid auflöst und das Rhodiumsalz als orangegelbes krystallinisches Pulver zurücklässt.

Bei unvorsichtigem Arbeiten, besonders bei Anwendung einer nicht ausreichenden Menge von salpetrigsaurem Alkali, bleibt leicht etwas Iridium bei den Sulfiden des Rhodiums-Platins und Rutheniums. Durch das Eintreten dieses Umstandes wird am Gange der Trennung des Rutheniums nichts geändert, doch ist man alsdann genöthigt die rückständigen Nitrite nochmals in Salzsäure zu lösen and die Behandlang mit salpetrigsaurem Natron und Schwefelnatrium zu wiederholen. Das Iridium geht dann in Lösung, während die Sulfide des Platins, Rhodiums und Rutheniums ausgefällt und, wie oben beschrieben, weiter getrennt werden.

Eine weitere Trennungsmethode gründet $\mathrm{Gibbs} *$ ) auf das Verhalten der Platinmetalle zu Luteokobaltchlorid. Hat man die Doppelchloride nach Verflüchtigung des Osmiums mit Chlorkalium and Salmiaklösung ausgewaschen, so werden sie zu feinem Pulver zerrieben mit $\mathrm{HO}$ gekocht. Man setzt vorsichtig, indem man die Flüssigkeit durch Soda neutral erhält, salpetrigsaures Kali hinzn, doch nicht mehr als eben zur Umwandlung des Iridiumchlorids in Sesquichlortirdoppelsalz erforderlich ist, filtrirt die olivengrüne Flüssigkeit $a b$ und wäscht mit wenig kaltem Wasser aus. Das Filtrat enthält neben nur wenig Kalium-Ruthen- und Kalium-Platinchlorid im Wesentlichen $3 \mathrm{KCl}+$ $\mathrm{Ir}_{2} \mathrm{Cl}_{3},-3 \mathrm{KCl}+\mathrm{Rh}_{2} \mathrm{Cl}_{3}$ und $2 \mathrm{KCl}+\mathrm{Ru}_{2} \mathrm{Cl}_{3}$.

Eine Lösung von Luteokobaltchlorid, welche man vorsichtig zusetzt, erzeugt einen blassledergelben Niederschlag von $6 \mathrm{NH}_{3} \mathrm{Co}_{2} \mathrm{Cl}_{3}+$ $\mathrm{Ir}_{2} \mathrm{Cl}_{3}$ und $6 \mathrm{NH}_{3} \mathrm{Co}_{2} \mathrm{Cl}_{3}+\mathrm{Rh}_{2} \mathrm{Cl}_{3}$, der zuerst mit siedendem Wasser und

*) Americ. Journ, of Science and Arts 37. 57; Journ. f. prakt. Chem. 94. 10 . 
nachher mit siedender verdünnter Salzsäure ausgewaschen wird, während alles Platin und Ruthen im Filtrate und Waschwasser enthalten ist. Filtrat und Waschwasser, in welchen sich ausser den leicht löslichen Luteokobaltdoppelchloriden des Ruthens und Platins auch noch Kaliumdoppelchloride des Ruthenchlorids und Sesquichlorürs finden, werden beinahe zur Trockne verdampft, mit starker Kalilauge gekocht, bis alles Ammoniak entwichen ist, darauf mit Salzsäure zur Trockene gebracht, das Chlorkobalt mit absolutem Alkohol extrahirt und endlich im Rückstande Platin und Ruthen nach dem oben angegebenen Verfahren getrennt.

Die unlöslichen Luteokobaltdoppelchloride des Iridiums und Rhodiums werden ebenfalls mit starker Kalilauge zersetzt. Der entstehende schwarze Niederschlag wird in Salzsäure gelöst, zur Trockene verdampft, mit Alkohol das Kobaltchlorid ausgekocht. Es bleiben die Doppelsalze $3 \mathrm{KCl}+\mathrm{Ir}_{2} \mathrm{Cl}_{3}$ und $3 \mathrm{KCl}+\mathrm{Rh}_{2} \mathrm{Cl}_{3}$ zurück; in diesen wird Iridium von Rhodium nach bekannter Methode getrennt.

Da die Ausführung dieser Methode die Anwendung grosser Mengen des schwer zu erlangenden Luteokobaltchlorids voraussetzt, so beschränkt der Verf. ihre Anwendung auf die Trennung des Rhodiums von Platin und Ruthen, welche in folgender Weise ausgeführt wird:

Die ausgewaschenen Doppelchloride werden in einem unglasirten Mörser sehr fein zerrieben, mit kleinen Mengen kalten Wassers so lange ausgewaschen als sich im Filtrat noch Ruthen nachweisen lässt. In der Lösung, welche die Chlorkaliumdoppelsalze von $\mathrm{RuCl}_{2}, \mathrm{Ru}_{2} \mathrm{Cl}_{3}$, $\mathrm{Rh}_{2} \mathrm{Cl}_{3}, \mathrm{IrCl}_{2}$ and wenig $\mathrm{KCl}, \mathrm{PtCl}_{2}$ enthält, wird das $\mathrm{IrCl}_{2}$ durch salpetrigsaures Natron in $\mathrm{Ir}_{2} \mathrm{Cl}_{3}$ verwandelt und darauf mit Luteokobaltchlorid gefällt. Man erhält einen Niederschlag von $6 \mathrm{NH}_{3} \mathrm{Co}_{2} \mathrm{Cl}_{3}+$ $\mathrm{Rh}_{2} \mathrm{Cl}_{3}$ mit wenig des entsprechenden Iridiumsalzes. Der Niederschlag wird abfiltrirt, mit Kalilauge zersetzt, mit Salzsäure zur Trockene gebracht und, nachdem der Kobaltchlorid durch absoluten Alkohol ausgekocht worden ist, verfährt man wie bei der Trennung des Iridiums von Ruthen. Im Filtrat von dem unlöslichen Rhodium- und Iridiumsalz trennt man Platin von Ruthen nach der oben beschriebenen Methode.

Da ein Theil des Ruthens im Filtrat als Kaliumruthensesquichlorür enthalten ist, dieses aber durch die salpetrige Säure reducirt werden könnte, so räth Gibbs dasselbe durch Kalilauge and Einleiten von Chlor in $\mathrm{KCl}+\mathrm{RuCl}_{2}$ überzuführen.

Der ledergelbe Niederschlag von $6 \mathrm{NH}_{3} \mathrm{CO}_{2} \mathrm{Cl}_{3}+3 \mathrm{IrCl}_{2}$, welcher durch Luteokobaltchlorid in einer Iridiumchloridlösung hervorgebracht wird, kann durch Kochen mit alkalischem Nitrit in das leichtlösliche Salz 
$6 \mathrm{NH}_{3} \mathrm{CO}_{2} \mathrm{Cl}_{3}+\mathrm{Ir}_{2} \mathrm{Cl}_{3}$ verwandelt werden, doch gelingt diese Umwandlung nur schwer vollständig bei Gegenwart von viel Kaliumplatinchlorid. Um Spuren von Iridium zu finden löst man daher das Platinsalz in heissem Wasser und fügt $6 \mathrm{NH}_{3} \mathrm{Co}_{2} \mathrm{Cl}_{3}+3 \mathrm{PtCl}_{2}$ hinzu, worauf sich nur das Iridiumsalz ausscheidet. Verf.. hält diess auch für die beste $\mathrm{Me}$ thode der quantitativen Trennug des Iridiums von Platin.

Zur Trennung der Sulfide des Rhodiums, Platins und Rutheniums (siehe pag. 135) schlägt der Verfasser in seiner letzten Mittheilung folgende einfachere Methode vor: Man erhitzt die getrockneten Sulfide in einem Porzellantiegel mit ihrem gleichen Gewicht einer Mischung aus 1 Theil salpetersaurem und 1 Theil kohlensaurem Baryt bis zum Hellrothglühen, extrahirt mit Salzsäure und schlägt den Baryt mit Schwefelsäure nieder ohne einen Ueberschuss derselben anzuwenden. Aus dem Filtrat fällt man durch Lateokobaltchlorid das unlösliche Rhodiumsalz und trennt in der Flüssigkeit Platin und Ruthen (nachdem das Kobalt entfernt ist) mittelst salpetrigsauren Kalis nach oben angegebener Methode.

Carey Lea befolgte zur Trennung der Gruppe der Platinmetalle im Allgemeinen das Verfahren von $\mathrm{Claus}$, indem er das californische Osmium und Iridium, welches in der Münze der vereinigten Staaten schon einmal mit Salpeter und Kali geschmolzen worden war, zunächst mit Königswasser kochte den Rückstand mit Salpeter und Kali schmelzte und diese Operation mehrmals wiederholte um das Osmium möglichst $z u$ entfernen. Zu diesem Zweck empiehlt C. Lea besonders anhaltendes Kochen mit Königswasser, während ein starker Luftstrom durchgeleitet wird. In der Lösung waren schliesslich Iridium, Ruthenium, Osmium, Rhodium und Platin enthalten, deren Trennung nach dem Verfahren von Cla us durch Fällen mit Salmiak and Auswaschen mit concentrirter, später mit verdünnter Salmiaklösung bewerkstelligt wurde. Er erhielt so einen Niederschlag (I), bestehend aus Iridiumsalmiak mit wenig Ruthenium, Osmium, Rhodium und Platin verunreinigt, eine Lösung (II), welche fast alles Ruthenchlorid enthielt, und eine verdünnte Salmiaklösung, in welcher sich noch kleine Mengen Iridium, Rhodium und Ruthen fanden.

Zur Reindarstellung des Iridiums bediente sich der Verfasser nun, abweichend von $\mathrm{Claus}$, der Reduction des Iridiumchlorids durch Oxalsäure. Die Oxalsäure verbält sich nämlich gegen die verschiedenen Platinmetalle, in Form ihrer höheren Chlorverbindungen, sehr ungleich. Während Iridchlorid durch Oxalsäure in der Kochhitze augenblicklich reducirt wird, widersteht das Ruthenchlorid sowie der Platinsalmiak 
ihrer Einwirkung lange Zeit, wenn sie auch nach langem Kochen gefällt resp. verändert werden.

Um das Iridium rein darzustellen löst $\mathrm{Car}$. L ea daher den Iridiumsalmiak (I) in dem 25fachen Gewicht Wasser und fügt nach und nach Krystalle von Oxalsäure hinzu. Wenn kein Aufbrausen mehr stattfindet, so kocht man 2-3 Minuten lang, sättigt halb mit Salmiak und stellt die Flüssigkeit in kaltes Wasser.

Der Platinsalmiak krystallisirt nach einigen Tagen heraus (war nicht aller $\mathrm{IrCl}_{2} \quad \mathrm{zu} \mathrm{Ir}_{2} \mathrm{Cl}_{3}$ reducirt, so krystallisirt natürlich auch etwas Iridiumsalmiak). Man führt nun durch Kochen init Königswasser das Sesquichlorür in Chlorid über, wonach beim Erkalten völlig ruthenfreier Iridiumsalmiak auskrystallisirt. Hat man viel Platin von wenig Iridium nach dieser Methode zu trennen, so ergibt sich ans dem oben angeführten Verhalten der Oxalsäure die Regel : eine hinreichende Wassermenge anzuwenden um alles Iridiumsalz in Lösung zu erhalten, und rechtzeitiges Beendigen des Kochens.

Maassanalytische Bestimmung des Schwefelarsens. Grä$\mathrm{ge}^{*}$ ) hat vorgeschlagen, gefälltes Schwefelarsen in der Ar maassanalytisch zu bestimmen, dass es mit Hülfe von reinem kohlensaurem Natron gelöst, zu der Lösung Stärkekleister und dann Jodlösung von bekanntem Gehalte gesetzt wird, bis bleibende Bläuung eintritt. Nach der angegebenen Gleichung (in welcher merkwürdiger Weise dem Schwefelarsen die Formel AsS beigelegt wird) sollen 5 Aeq. Jod auf 1 Aeq. Arsen verbraucht werden, also etwa:

$$
\mathrm{AsS}_{3}+5 \mathrm{~J}+5 \mathrm{HO}=\mathrm{AsO}_{5}+5 \mathrm{HJ}+3 \mathrm{~S} .
$$

Wenn man die Mittheilungen des Verfassers liest, kommt man zu der Meinung, die Reaction verlaufe ganz glatt nnd lasse einen überaus hohen Grad von Genauigkeit erreichen, denn von den mitgetheilten Versuchen stimmen die beiden ersten mit der Berechnung absolut, der vierte weicht nur am $0,05 \mathrm{CC}$. 1/10 Jodlösung ab und nur beim dritten findet sich eine Abweichung von 0,2 CC. $1 / 10$ Jodlösung, - stellt man aber einige Versuche an, so kommt man zu ganz anderen Resultaten. Zunächst ergibt sich, dass der Endpunkt überaus schwer oder richtiger gar nicht mit Sicherheit angegeben werden kann. Bei einem ersten Versuch erforderten $10 \mathrm{CC}$. einer verdünnten, mit Hülfe von reinem, zur Lösung nur eben genügendem kohlensaurem Natron bereiteten Auflösung von reinem Schwefelarsen

*) Journ. f. prakt. Chem. 96. 261. 